\title{
Raised serum APRIL levels in patients with systemic lupus erythematosus
}

\section{T Koyama, H Tsukamoto, Y Miyagi, D Himeji, J Otsuka, H Miyagawa, M Harada, T Horiuchi}

Objective: To determine whether serum levels of a proliferation-inducing ligand (APRIL) are raised in patients with systemic lupus erythematosus (SLE) and correlate with autoantibody titres or disease activity, or both.

Methods: Serum samples from 48 patients with SLE, 41 normal healthy subjects, and 21 patients with rheumatoid arthritis (RA) were assayed for APRIL by enzyme linked immunosorbent assay. Medical charts were retrospectively reviewed for autoantibody titres and immunoglobulin levels. Disease activity was assessed using the British Isles Lupus Assessment Group (BILAG) index.

Results: The APRIL levels in the serum samples from patients with SLE were significantly higher than in those from healthy controls and those from patients with RA. Serum APRIL levels did not correlate with serum lgG and IgM levels, but had a tendency to correlate with anti-double stranded DNA antibody titres. Moreover, serum APRIL levels correlated significantly with musculoskeletal manifestations among patients with SLE when assessed by the BILAG index.

Conclusion: APRIL may be an important factor in raised autoantibody titres and musculoskeletal disease in patients with SLE. Patients with raised serum APRIL levels may be ideal candidates for therapeutic targeting of APRIL.

A proliferation-inducing ligand (APRIL, also called TNFSF13), a newly identified member of the tumour necrosis factor ligand family, is a type II membrane binding protein of 250 amino acids. ${ }^{1}$ Originally, APRIL was reported to have a regulatory role in tumour growth. ${ }^{1}$ APRIL is a close sequence homologue of the recently reported B cell activation factor (BAFF, also known as BlyS/zTNF4/TALL-1), also a member of the tumour necrosis factor family. ${ }^{2}$ BAFF promotes B cell differentiation, proliferation, and survival of a subset of immature B lymphocytes, and BAFF transgenic mice develop a lupus-like phenotype characterised by high titres of anti-DNA antibodies, hypergammaglobulinaemia, and glomerulonephritis. ${ }^{3}$ In APRIL transgenic mice, $\mathrm{T}$ cell survival and antigen-specific antibody responses are enhanced. ${ }^{4}$

APRIL binds to two of the three BAFF receptors (B cell maturation antigen (BCMA) and transmembrane activator and CAML interactor (TACI)) and is supposed to have a regulatory role in B cell proliferation. ${ }^{5}$ The treatment of lupus prone $(\mathrm{NZB} \times \mathrm{W}) \mathrm{Fl}$ mice with a soluble TACI-immunoglobulin fusion protein (soluble decoy receptor for BAFF and APRIL) inhibits the development of proteinuria and prolongs survival of the animals. ${ }^{6}$ It has also been reported that gene polymorphism of APRIL is associated with systemic lupus erythematosus (SLE). ${ }^{7}$ These findings indicate that APRIL as well as BAFF may be involved in the development of SLE. The serum levels of BAFF are raised in patients with SLE, Sjögren's syndrome, and rheumatoid arthritis (RA). ${ }^{8}$ Recently, increased levels of BAFF and APRIL were reported in the synovial fluid of inflamed joints. ${ }^{9}$ However, serum levels of APRIL in patients with SLE have not been reported.

In this article, we report for the first time increased serum APRIL levels in patients with SLE. Our results indicate that raised levels of serum APRIL may be an important factor in the pathogenesis of SLE.

\section{PATIENTS AND METHODS}

\section{Subjects}

Peripheral blood was obtained from 48 patients ( 46 female, 2 male, mean age 37.9 years) meeting the American College of Rheumatology (ACR) criteria for the classification of SLE. Twenty one patients with RA ( 18 female, 3 male, mean age 55.7 years) met the ACR criteria for RA and were followed up by Kyushu University Hospital. Forty one control subjects (33 female, 8 male, mean age 35.1 years) were healthy volunteer donors who were recruited from Kyushu University Graduate School of Medical Sciences personnel. Informed consent was obtained from each patient and control. All were Japanese and their serum samples were stored at $-20^{\circ} \mathrm{C}$ until use.

\section{ELISA for measurement of APRIL}

The collected serum samples were assayed for APRIL by an antigen-capture enzyme linked immunosorbent assay (ELISA). ELISA plates (Becton Dickinson Labware, Franklin lakes, NJ) were coated with $5 \mu \mathrm{g} / \mathrm{ml}$ rabbit antihuman APRIL polyclonal antibody (ED2; ProSci Inc, Poway, CA) overnight at $4^{\circ} \mathrm{C}$. After non-specific binding had been blocked with $1 \%$ bovine serum albumin-phosphate buffered saline, the collected serum samples were added, followed by the detection antibody, goat antihuman APRIL antibody (Genzyme Techne, Minneapolis, MN). Rabbit antigoat IgG horseradish peroxidase (MBL, Nagoya, Japan) and 2,2'-azino-bis(3-ethylbenzothiazoline-6-sulphonic acid) diammonium salt (Wako Pure Chemical Industries, Osaka, Japan) were used for detection. Enzyme activities were read at an optical density of $405 \mathrm{~nm}$, and a standard curve was generated using recombinant human APRIL (Genzyme Techne). Immunoprecipitation of the APRIL was performed by adding BCMA-FC chimera (Genzyme Techne), soluble receptor for APRIL, to the serum followed by addition of protein A-Sepharose (Sigma-Aldrich, St Louis, MO). Recombinant human BAFF was purchased from Genzyme Techne.

Abbreviations: ACR, American College of Rheumatology; APRIL, a proliferation-inducing ligand; BAFF, B cell activation factor; BCMA, B cell maturation antigen; BILAG, British Isles Lupus Assessment Group; ELISA, enzyme linked immunosorbent assay; RA, rheumatoid arthritis; SLE, systemic lupus erythematosus; SLEDAI, Systemic Lupus

Erythematosus Disease Activity Index; TACl, transmembrane activator and CAML interactor 


\section{Measurement of autoantibody and assessment of the disease activity}

The serum levels of anti-dsDNA and anti-Sm antibody were measured by an ELISA kit (MBL) according to manufacturer's instructions. We evaluated the disease activity at the time of the serum sampling using the British Isles Lupus Assessment Group (BILAG) index and the Systemic Lupus Erythematosus Disease Activity Index (SLEDAI).${ }^{10}$ The BILAG index has been validated and shown to be reproducible and reliable. ${ }^{11}$ The index allocates separate scores to each of eight organ based systems.

\section{Statistical analysis}

All statistical analyses were performed using StatView software (Abacus concept Inc, Cary, NC). Non-parametric testing was performed by Mann-Whitney's U test between two groups. Correlation was determined by Pearson's product-moment correlation for interval data or by Spearman's rank order correlation for ordinal data or for interval data that did not follow normal distribution. When we investigated the correlation of BILAG with serum APRIL levels, we used 4, 3, 2, I instead of A, B, C, D of the BILAG index score, respectively.

\section{RESULTS}

Raised serum levels of APRIL in the patients with SLE We measured the serum levels of APRIL in 48 patients with SLE, 21 patients with RA, and in 41 healthy controls using a sandwich ELISA. The mean (SD) serum levels of APRIL in patients with SLE (84.1 (106.1)) were significantly higher than those in the normal cohorts $(16.0(25.6), p=0.0004)$ and in patients with RA (25.1 (34.5), p=0.046), while those in patients with RA were no higher than those in normal controls (fig 1). The serum APRIL levels in most of the normal controls were $<50 \mathrm{ng} / \mathrm{ml}$, and only $3 / 41$ (7\%) controls had levels $>100 \mathrm{ng} / \mathrm{ml}$. In contrast, the APRIL levels in the majority of patients with SLE were $>50 \mathrm{ng} / \mathrm{ml}$, and 20/48 $(42 \%)$ patients had levels $>100 \mathrm{ng} / \mathrm{ml}$. Immunoprecipitation of the serum samples with BCMA-Fc chimera significantly decreased the APRIL level by this ELISA system. In addition, recombinant human BAFF was not detected by this ELISA (data not shown). This result suggested the specificity of our ELISA system for APRIL.

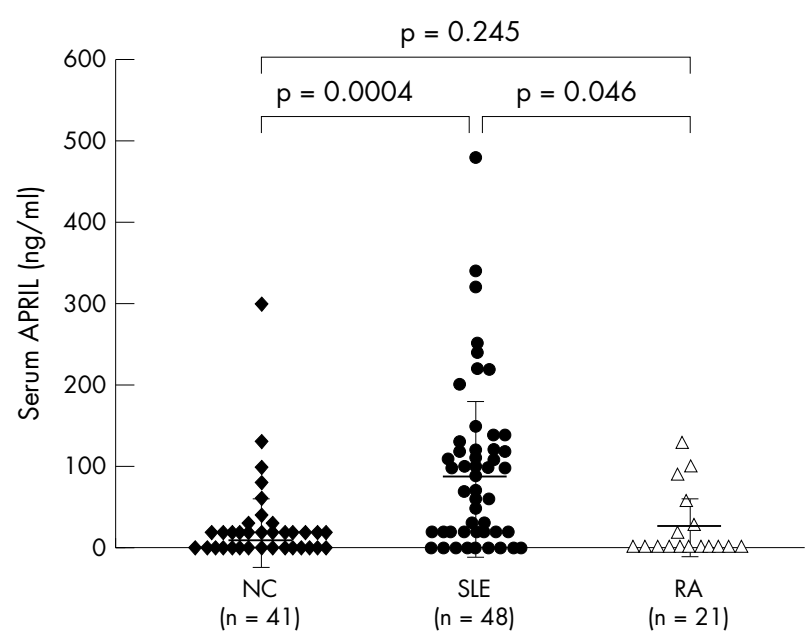

Figure 1 Serum levels of APRIL in the 48 patients with SLE were significantly higher than those in 41 healthy controls $(p=0.0004)$.

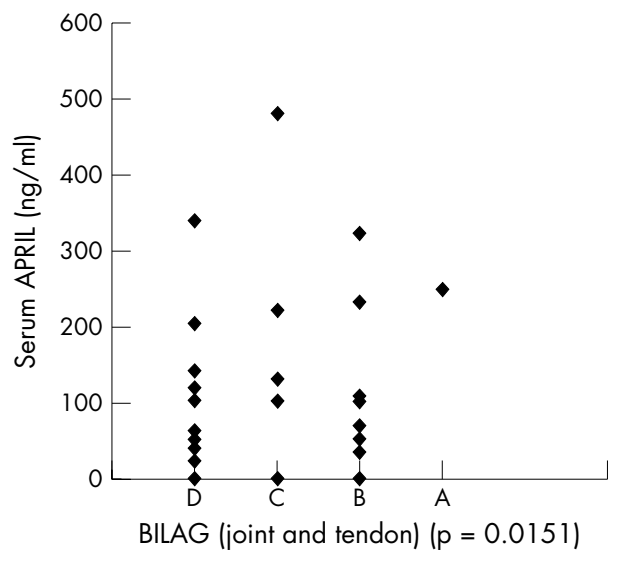

Figure 2 Correlation between the serum APRIL levels and musculoskeletal manifestations among patients with SLE. The correlation coefficient was determined by Spearman's rank order correlation.

\section{Correlation between serum APRIL levels and raised autoantibody titres}

A retrospective chart review showed that 45 patients with SLE had been screened for serum anti-dsDNA antibodies in the same blood collected for serum APRIL determination. The anti-dsDNA antibody titres had a tendency to correlate $\left(r_{\mathrm{s}}=0.277, \mathrm{p}=0.065\right)$ with serum APRIL levels. The serum levels of APRIL did not correlate with IgG, IgM, or anti-Sm antibody titres in patients with SLE. There was no correlation between serum levels of APRIL and the dose of corticosteroids or immunosuppressant drugs.

\section{Correlation between serum APRIL levels and disease activity}

We also analysed the clinical data of the patients with SLE using the BILAG index (fig 2). The serum APRIL levels correlated significantly with the BILAG index score of musculoskeletal disease $(p=0.0151)$, but there was no correlation with score for the the other seven organs, including the nervous system and the kidney. Additionally, no correlation was found between the serum APRIL level and the SLEDAI.

\section{DISCUSSION}

In this study we showed for the first time that APRIL levels were raised in serum samples from patients with SLE and were associated with a phenotype of SLE.

SLE is a prototypic systemic autoimmune disease characterised by the production of autoantibodies against a spectrum of nuclear antigens. ${ }^{12}$ These autoantibodies are produced by autoreactive B lymphocytes in the presence or absence of autoreactive $\mathrm{T}$ lymphocytes. ${ }^{12}$ APRIL has a regulatory role in B cell proliferation, ${ }^{5}$ and affects the $\mathrm{T}$ cell response, ${ }^{4}$ and dendritic cells induced CD40 independent immunoglobulin class switching through APRIL. ${ }^{13}$ Furthermore, the soluble decoy receptors for APRIL prolong the survival of lupus prone $(\mathrm{NZB} \times \mathrm{W}) \mathrm{F} 1$ mice. ${ }^{6}{ }^{14}$ These lines of evidence suggest that increased serum APRIL may play a part in the pathogenesis of SLE. The mechanism of the increased serum APRIL levels might be explained as follows. APRIL is synthesised by monocytes and dendritic cells in response to interferons. Because type I interferon regulated genes are up regulated in peripheral blood mononuclear cells from patients with SLE, raised APRIL could be explained by the interferon-induced expression in these cell types. ${ }^{13}$ Another possible mechanism for raised serum APRIL levels in SLE is that there may be polymorphisms in the promoter region of the APRIL gene, and these might affect gene expression. 
Serum APRIL levels tended to correlate with anti-dsDNA antibody titres $(p=0.065)$. Recombinant APRIL costimulates $B$ cells in vitro and in vivo, although its potency is less than that of BAFF. ${ }^{5}$ It is thus suggested that APRIL may be an important factor in raised autoantibody titres.

It is noteworthy that serum APRIL levels correlated significantly with the musculoskeletal manifestations of SLE, mostly arthritis, in the present study. It has been reported that joint inflammation and joint destruction of collagen-induced arthritis, a model of non-infectious inflammatory arthritis, are inhibited by the treatment with TACIimmunoglobulin, an antagonist of APRIL and BAFF. ${ }^{15}$ Recently, it was reported that the levels of APRIL and BAFF are increased in the synovial fluid of inflamed joints, but not in the serum, indicating increased local production of BAFF and APRIL.' Because B cells have a role in activation and cytokine production of $\mathrm{T}$ cells in joints, prolonged survival or activation of B cells by APRIL may lead to joint inflammation. Although we could not analyse the APRIL levels in synovial fluid from patients with SLE, an increase of APRIL may lead to the worsening of arthritis in patients with SLE.

Our findings suggest that an APRIL antagonist may be a suitable treatment for patients with SLE. Further studies on the regulation of the APRIL gene in patients with SLE, and the association of serum APRIL with other autoimmune diseases, are warranted.

\section{ACKNOWLEDGEMENT}

This work was supported by a grant in aid from the Ministry of Education, Science, Sports and Culture of Japan (14570415).

\section{Authors' affiliations Japan \\ Correspondence to: $\mathrm{Dr} \mathrm{H}$ Tsukamoto, tsukamot@intmed1.med.kyushu-u.ac.jp}

T Koyama, H Tsukamoto, Y Miyagi, D Himeji, J Otsuka, H Miyagawa, M Harada, T Horiuchi, Medicine and Biosystemic Science, Kyushu University Graduate School of Medical Sciences, Fukuoka 812-8582,
Accepted 27 November 2004

Published Online First 2 December 2004

\section{REFERENCES}

1 Hahne M, Kataoka T, Schroter M, Hofmann K, Irmler M, Bodmer JL, et al. APRIL, a new ligand of the tumor necrosis factor family, stimulating tumor cell growth. J Exp Med 1998;188:1185-90.

2 Schneider P, Mackay F, Steiner V, Hofmann K, Bodmer JL, Holler N, et al. BAFF, a novel ligand of the tumor necrosis factor family, stimulates B cell growth. J Exp Med 1999; 189:1747-56.

3 Mackay F, Woodcock SA, Lawton P, Ambrose C, Baetscher M, Schneider P, et al. Mice transgenic for BAFF develop lymphocytic disorders along with autoimmune manifestations. J Exp Med 1999;190:1697-710.

4 Stein JV, Lopez-Fraga M, Elustondo FA, Carvalho-Pinto CE, Rodriguez D, Gome-Caro R, et al. APRIL modulates B and T cell immunity. J Clin Invest 2002; 109:1587-98

5 Marsters SA, Yan M, Pitti RM, Haas PE, Dixit VM, Ashkenazi A. Interaction of the TNF homologues BLyS and APRIL with the TNF receptor homologues BCMA and TACI. Curr Biol 2000;10:785-8.

6 Gross JA, Johnston J, Mudri S, Enselman R, Dillon SR, Madden K, et al. TACI and BCMA are receptors for a TNF homologue implicated in B-cell autoimmune disease. Nature 2000;404:995-9.

7 Koyama T, Tsukamoto H, Masumoto K, Himeji D, Hayashi K, Harada M, et al. A novel polymorphism of the human APRIL gene is associated with systemic lupus erythematosus. Rheumatology (Oxford) 2003;42:980-5.

8 Zhang J, Roschike V, Baker KP, Wang Z, Alarcon GS, Fessler BJ, et al. A role for B lymphocyte stimulator in systemic lupus erythematosus. J Immunol 2001;166:6-10.

9 Tan SM, Xu D, Roschke V, Perry JW, Arkfeld DG, Ehresmann GR, et al. Local production of $B$ lymphocyte stimulator protein and APRIL in arthritic joints of patients with inflammatory arthritis. Arthiritis Rheum 2003;48:982-92.

10 Bonbardier C, Gladman DD, Urowitz MB, Caron D, Chang CH. Derivation of the SLEDAl: a disease activity index for lupus patients. The committee on prognosis studies in SLE. Arthritis Rheum 1992;35:630-40.

11 Hay EM, Bacon PA, Gordon C, Isenberg DA, Maddison P, Snaith ML, et al. The BILAG index: a reliable and valid instrument for measuring clinical disease activity in systemic lupus erythematosus. Q J Med 1993;86:447-58.

12 Kotzin BL. Systemic lupus erythematosus. Cell 1996;85:303-6.

13 Litinskiy MB, Nardelli B, Hilbert DM, He B, Schaffer A, Casali P, et al. DCs induce CD40-independent immunoglobulin class switching through BlyS and APRIL. Nat Immunol 2002;3:822-9.

14 Rennert P, Schneider P, Cachero TG, Thompson J, Trabach L, Hertig S, et al. A soluble form of $B$ cell maturation antigen, a receptor for the tumor necrosis factor family member APRIL, inhibits tumor cell growth. J Exp Med 2000;192:1677-83

15 Wang H, Marsters SA, Baker T, Chan B, Lee WP, Fu L, et al. TACI-ligand interactions are required for $T$ cell activation and collagen-induced arthritis in mice. Nat Immunol 2001;2:632-7. 